\title{
ETSI ACTIVITIES ON NEXT GENERATION NETWORKS
}

The contribution deals with the current activities in ETSI (European Telecommunications Standards Institute) on Next Generation Networks (NGN). The first part includes the general information on ETSI and the creation of the new Technical Committee TISPAN (Telecommunications and Internet converged Services and Protocols for Advanced Networks) which is responsible for all aspects of the wired networks like signalling protocols or systems interworking and which is currently moving towards definition and specification of the NGN. The next part is aimed at the current structure of the TC TISPAN and finally, the key objectives within the TISPAN Releases are presented.

The European Telecommunications Standards Institute (ETSI) is an independent, non-profit organization, whose mission is to produce telecommunications standards for today and for the future.

Based in Sophia-Antipolis in the south of France, ETSI unites 688 members from 55 countries, and brings together manufacturers, network operators and service providers, administrations, research bodies and users - providing a forum in which all key players can contribute.

ETSI's Members determine the Institute's work programme, allocate resources and approve its deliverables. As a result, ETSI's activities are closely aligned with market needs and there is wide acceptance of its products. ETSI's standards are built on consensus.

ETSI is structured in different working groups called Technical Committee (TC). Each of them is responsible for a given technical area like: Satellite, Radio Communications, Fixed Networks Communications, Safety, and Mobile Communications.

One of them, ETSI Technical Committee TISPAN (Telecommunications and Internet converged Services and Protocols for Advanced Networks) was created in September 2003 as the result of the combination of TC SPAN (Services and Protocols for Advanced Networks) and ETSI Project TIPHON (Telecommunications and IP Harmonization Over Networks). The decision to merge the two former bodies has been taken by the ETSI Board in view of maximizing the synergies between the top down approach of TIPHON in their definition of technology independent features network architecture to support service capabilities and the bottom up approach followed by SPAN in their workplan to produce their specifications enabling a smooth evolution of legacy networks towards IP-based networks to support a wide range of new generation services. The objective of TISPAN is indeed to produce a first set of specifications that are awaited by the ETSI members in order to provide a confident basis for the development and implementation of first steps of NGN systems supporting truly innovative multimedia services.
During the first half of 2003, EP TIPHON advanced significantly the support of multiple media applications, providing extended security features, user authentication and information confidentiality. These efforts were aimed at maximizing the service capabilities and network features harmonization with other ongoing standards developments, particularly with the Universal Mobile Telecommunications System (UMTS ${ }^{\mathrm{TM}}$ ) under the auspices of the Third Generation Partnership Project (3GPP ${ }^{\mathrm{TM}}$ ). In addition plans for a proposed activity were discussed in order to further enhance the set of fundamental service capabilities (such as presence management, messaging) aiming at enabling ubiquitous multimedia communications.

Concurrently, up to summer 2003, TC SPAN continued to actively contribute to the activities of the International Telecommunication Union Telecommunications Standardization Sector (ITU-T), and to work jointly with the Third Generation Partnership Project (3GPPTM) particularly for the development of interfaces with service creation environment and application platforms through Application Programming Interfaces (APIs) providing Open Services Access (OSA). These sustained activities aimed at ensuring that fixed networks requirements are well accommodated by the emerging network architectures and technologies.

Combining both helped strengthen synergies and confirmed the ETSI crucial role in rolling out Multi-service/Multimedia Network technologies in Next Generation Networks (NGN) environments at the global level.

TC TISPAN is responsible for all aspects of the wired networks like signalling protocols or systems interworking. It is currently moving towards definition and specification of the Next Generation Networks (NGN). Its architectural concept is based on a 3-level structure: Service, Control and Transport. Access to NGN shall be widely open. The first approach is the one defined for the 3GPP Release 6 Architecture called IP Multimedia Services (IMS). This common approach will ease the fixed-mobile convergence, which will allow the seamless service goal. TC TISPAN has defined a so-called NGN Project with a very ambitious aim,

\footnotetext{
* Christian Julien

European Telecommunications Standards Institute, Secretariat, ETSI, 650, Route des Lucioles, Sophia Antipolis, France,

E-mail: Christian.julien@etsi.org
} 
which is to produce a first set of specifications by the end of June 2005.

TISPAN held its kick-off meeting in September, bringing together the TIPHON and SPAN cultures and expertise, and combining their respective top-down and bottom-up approaches. The first set of NGN specifications for the support of a realistic set of high priority real-time conversational multimedia services and content delivery services (e.g. Video on demand, TV program distribution) in addition to Internet-based applications is expected in the year 2005 timeframe.

Its second meeting held in December resulted in the launch of the TISPAN_NGN project, taking a pragmatic phased approach. The scope of TISPAN_NGN Release 1 was initially discussed and consensus was reached to adopt the 3GPP (SIP-based) IP Multimedia Subsystem (IMS) as a component basis for the support of NGN multimedia conversational services. It is planned to work in cooperation with the $3 \mathrm{GPP}$ in order to define the IMS adaptations required for the support of the XDSL IP-Connectivity access network technology. The plan is to complete TISPAN-NGN Release 1 by mid-2005 according to the Telecom industry expectations.

Its third meeting held in March 2004 resulted in the consolidation of services to be included in the NGN Release 1 and in the decision to prepare a first joint session with the 3GPP relevant groups (Service Access (SA) and Core Network (CN)). A date has been agreed on 22-23 June 2004. During this common session overview of organisations, basic rules and working methods will be presented and a way to progress together will be specified.

An aggressive TISPAN work plan is being set-up, with the hope that the ambitious goal can be met to enable initial NGN deployment steps, while aiming at smooth fixed and mobile networks convergence in terms of features supported, architectures deployed and service capabilities provided. That's a lot of work ahead of the TISPAN participants; let us be part of this great challenge of contributing to the definition of the 21st century telecom network! But what is TISPAN and how is it organised to complete successfully this task?

TISPAN is structured into 7 Working Groups (WG) dealing mainly with 5 projects. Each WG is chaired by a WG chairman and each project is under the responsibility of a Project leader. WGs are: Services, Architecture, protocols, Numbering/Addressing/ Routing (NAR), Quality of Service (QoS), Testing, Security and Network Management. Five projects are: Next Generation Network (NGN), Open Service Access (OSA) Dynamic synchronous Transfer Mode (DTM), Emergency Telecommunications (EMTEL) and Fixed-Multimedia Messaging Service (F-MMS).

WG1 on Services is responsible for:

- Conducting studies leading to deliverables on new generation services and applications (from the user perspective);

- Co-ordination and preparation of service descriptions for NGN, including:

- Inter-personal real-time services,
- Video (on-demand, streaming, unicast and broadcast),

- Data (Storage, processing), Messaging

- Multimedia and group communication

- For different market segments and communities.

- Identification of generic NGN functional requirements to support service operation.

WG2 on Architecture is responsible for:

- Network Intelligence, Universal personal and terminal Mobility, NGN architectures and Home Environment functional and architectural requirements; including the definition and the use of an overall functional architecture;

- Standards development activities related to Network architecture and its evolution, including inter-working and IP matters at the service, transport and control level;

- Studies on the functions, and the reference points required for inter-working between the emerging new architecture networks and legacy networks (IN/ISDN/PSTN);

- Definition and analysis of Functional Entities, Message Sequences and Information Elements;

- Studies on the functional requirements that Service Providers have when accessing the networks of Public Network Operators (PNO) in the fulfilment of the Special Network Access directives of the European Union (EU); including functional interfaces for Third Party Service APIs;

- Studies in the field of third generation mobility systems (e.g.: IMT-2000 and UMTS) concerning inter-working with existing and emerging IP-based Core Networks. This includes Interaccess-system inter-operability, mobility and registration.

WG3 on Protocols is responsible for:

- Defining all aspects of Protocols: protocol definition, protocol requirements, protocol mapping, protocol profiles, analysis of protocols developed by other bodies, protocol extensions and inter-working specifications.

WG4 on NAR is responsible for:

- Conducting studies leading to deliverables on: numbering, naming, addressing and routeing;

- Addressing and Naming translation;

- Representing ETSI at the ENF (European Numbering Forum);

- Collaborating with ERO (the European Radio Office) on European numbering, naming and addressing issues;

- Representing ETSI at the ETNS Steering Committee.

WG5 is responsible for:

- Conducting studies leading to deliverables on:

a) application- and user-centric quality of service (QoS) parameters, objectives and measurement methods,

b) network-centric performance (NP) parameters, objectives and measurement methods necessary to meet the identified QoS objectives, and

c) determining the requirements of the protocols necessary to allocate and manage network resources to meet the identified QoS\&NP objectives,

for packet-based, circuit-switched and hybrid networks supporting multimedia services. 
WG6 is responsible for:

- Management and co-ordination of the development of the testing specifications for the next generation telephony;

- Providing testing specifications for TISPAN-developed specifications and profiles;

- Maintaining existing testing specifications as required;

- Tracking ongoing worldwide bake-off, interoperability, testing and certification activities of interest to TISPAN.

WG7 is responsible for:

- Conducting studies leading to deliverables on Security;

- Management and co-ordination of the development of security specifications for the next generation telephony and multimedia communications;

- Investigation of security services and mechanisms required for providing services over the Internet;

- Development of security analyses of candidate protocols and network elements to be used within the NGN framework to implement capabilities e.g., EMTEL aspects, IPv6 migration, keying strategies and methods;

- Tracking ongoing worldwide security activities of interest to TISPAN.

WG8 is responsible for:

- Developing a consistent and harmonised approach to Telecommunications Management across all disciplines and technologies under the umbrella of the ETSI Standardisation activities;

- Telecommunications Management encompasses the management of all types of telecommunication equipment, networks, services and although biased to the Telecommunications Management Network (TMN)

So far the applied TISPAN working method is to have 4 Plenary a year where all WGs work together for progressing their relevant studies. Between each Plenary usually there is an interim meeting more dedicated to any Project where the Project activity is fasten. All WG chairmen and Project leaders met regularly into the TISPAN Management group for coordinating their respective activities either by special meeting or by regular conference call. Relationship with the other bodies is maintained by a standard liaison statement procedure.

After describing TC TISPAN, let's add some words on the TISPAN-NGN project which is the purpose of this paper.

From a technical point of view, TISPAN-NGN can be considered as a multi-service/multimedia network based upon the use of packet switching technologies. Architecturally speaking, it can be envisioned as consisting of a packet based core network with interfaces to several different access networks (e.g. xDSL / Wireless LAN etc) possibly using different underlying transport technologies.

From an application viewpoint, TISPAN-NGN release \#1 supports two broad types of application:

- Session based: Examples include conversational applications such as VoIP/Multimedia calls and on-line gaming as well as non-real-time conversational applications such as messaging.
- Non-session based: Examples include streaming applications such as web-casting / broadcasting and video and audio/music on demand.

While TISPAN-NGN supports the co-existence of both session and non-session based applications on a single NGN, in the relevant standards these applications are modelled as being provided through logically separate sub-systems.

A key assumption within TISPAN-NGN is that services themselves are not standardized. Instead TISPAN-NGN defines service capabilities. In such network operators are free to combine service capabilities (potentially provided by 3 rd parties) to offer specific services to subscribers. Examples of service capabilities include presence, location, non-real time data transfer (e.g. email, ftp), web-browsing, authorization mechanisms supporting single signon, and the capability to set up sessions or streaming media with particular characteristics in support of offering conversational and/or streaming based services.

Another important activity relevant to NGN has already been initialised within Network Management group with the aim to specify at least for the NGN Release 1, the new NGN Operating Sub System (OSS). In order to prepare this task a new Specialist Task Force (STF) has been started end of March 2004 with the active participation of Ms. T. Kovacikova from University of Zilina, Slovakia. The purpose of this group of experts is to investigate the current status of the standardization in the domain of Network Management and to identify where the lack of standards for completing the work is.

As stated today within the content of Release 1, amongst the different scenarios envisaged for introducing the NGN within the current country there are:

- PSTN/ISDN replacements,

- PSTN/ISDN interworking,

- Cable network interworking,

- $3 \mathrm{GPP}$ interworking,

- Internet interconnection.

Also included into Release 1 the following network accesses are envisaged:

- ADSL,

- WLAN,

- Cable network,

- Corporate networks,

- UMTS

The content of the further Releases is not yet known in detail but integration of IPv4 into IPv6 or the full Mobility will certainly be the main issues.

The current agreed schedule for the different Releases is as follows:

- Release 1: June 2005,

- Release 2: December 2006,

- Release 3: June 2008. 
Finally some figures for sizing TC TISPAN:

- Currently its work programme is made of about 142 work items, 16 standards and/or technical reports have already been published;

- There are 14 exploder lists in which more than 600 delegates coming from every part of the world are registered.
- Usual amount of input contributions per Plenary is more than 200 documents;

- More than 100 delegates attend usually any Plenary. 